\title{
Test and Measurement Methods for Fuel Cell Technology
}

\author{
Denis Kramer ${ }^{a \star}$, Augustin J. McEvoy ${ }^{\mathrm{b}}$, Ingo Schneider ${ }^{\mathrm{a}}$, Holger Kuhn ${ }^{\mathrm{a}}$, Alexander Wokaun ${ }^{\mathrm{a}}$, and \\ Günther G. Scherer ${ }^{\mathrm{a}}$
}

\begin{abstract}
Test and measurement methods, particularly electrochemical methods, applicable to fuel cell technology are reviewed. The difficulties of application of diagnostic tools and interpretation of results are highlighted. Specific requirements of the different fuel cell types, both high and low temperature variants, are dealt with. Finally, recently developed in situ imaging methods and their diagnostic significance are presented.
\end{abstract}

Keywords: Diagnostics · Fuel cell · Impedance spectroscopy · Neutron imaging $\cdot$ Reference electrode

\section{Introduction}

Without measurement and test procedures no technology is possible, let alone advanced or innovative engineering and applications. Diagnostics are key elements in research, development, demonstration, and production for the validation of basic theoretical concepts, the selection and qualification of materials, the evaluation of device and system structures, then production and installation as well as economy, efficiency and reliability in service. The test and diagnostic procedures must identify the key parameters for functionality of the device, as well as address degradation and failure mechanisms. Since fuel cells comprise advanced materials electrodes, electrolytes, interface catalysts and fuel processing systems - in an electrochemical environment, the appropriate testing procedures involve the physicochemical parameters of solid state materials, but also and particularly the electrical and electrochemical behaviour of those materials and their interfaces. However, since materials sci-

${ }^{\star}$ Correspondence: D. Kramera

Tel.: +41563104140

Fax: +41563104415

E-Mail: denis.kramer@psi.ch

aElectrochemistry Laboratory

General Energy Department

Paul Scherrer Institut

$\mathrm{CH}-5232$ Villigen PSI

bInstitut des Sciences et Ingénierie

de la Chimie (ISIC-FSB)

Ecole Polytechnique Fédérale de Lausanne (EPFL)

$\mathrm{CH}-1015$ Lausanne ence test and measurement procedures are widely applied in electrical and mechanical engineering practice, as well as in relevant research and development, this review will concentrate on the electrochemical procedures.

\section{Principles of Fuel Cell Testing}

Since any electrochemical cell requires at least three components, the two electrodes and the electrolyte, test and measurement on the device as a whole is inadequate to distinguish the processes occurring at each of the two electrode-electrolyte interfaces. In consequence, the information necessary on which to base either diagnostics of limiting processes and degradation, or a strategy for materials and interface development and optimisation, is concealed. It should also be recognised that an electrochemical cell is a closely coupled system, and any modification of a single component can influence the functionality of other components or of the cell as a whole. Measurement of the current across a cell as a function of the potential difference is therefore indicative of the performance of the system, but the partition of loss mechanisms, e.g. electrocatalytic effects or mass transport limitations, is not accessible. Hence, an approach is needed that is capable of isolating and measuring the effects at a single electrode-electrolyte interface. One such approach is the introduction of a third electrode. This electrode ideally provides a stable and well-defined potential, due to a fast and reversible reaction at its interface - such as oxygen reduction at atmospheric pressure (in the case of a solid oxide fuel cell) or hy- drogen oxidation (in the case of polymer electrolyte fuel cells). The potential is sensed by an electronic system whose input impedance is so high (>10 G $\Omega$ ) that negligible current can be drawn. Consequently, the electrode remains unpolarised and no overpotential effect is induced. This reference electrode channel is then used to measure or control the potential of the working electrode under test. The three most common three-electrode arrangements are sketched in Fig. 1. It must be recognised that a systematic error may be introduced for the setups a) and c), since the reference electrode senses the potential of the electrolyte at its point of contact, not necessarily on an equipotential plane between the working and counter electrodes. Precise measurements therefore require a correction (cf. Fig. 8), which can be most difficult in the case of state-of-the-art thin electrolyte devices [1]. The arrangement shown in Fig. 1b circumvents this problem, but since the sensing point is placed inside the electrolyte, the potential of the reference is not strictly defined. Note that the test electrode has been referred to as the working electrode rather than anode or cathode, as the setup can function in all four quadrants of the current/voltage plane, and therefore accommodate both anodic and cathodic processes at whatever potential is demanded for the given electrochemical reaction taking place. Furthermore, it has to be stated that such an experimental setup is ideally applied to simple (homogeneous over the active area) systems.

A variety of standard analytical electrochemical methods can be applied to fuel cells, either in the three or two electrode configuration. The three electrode configuration is preferred in order to investigate processes 


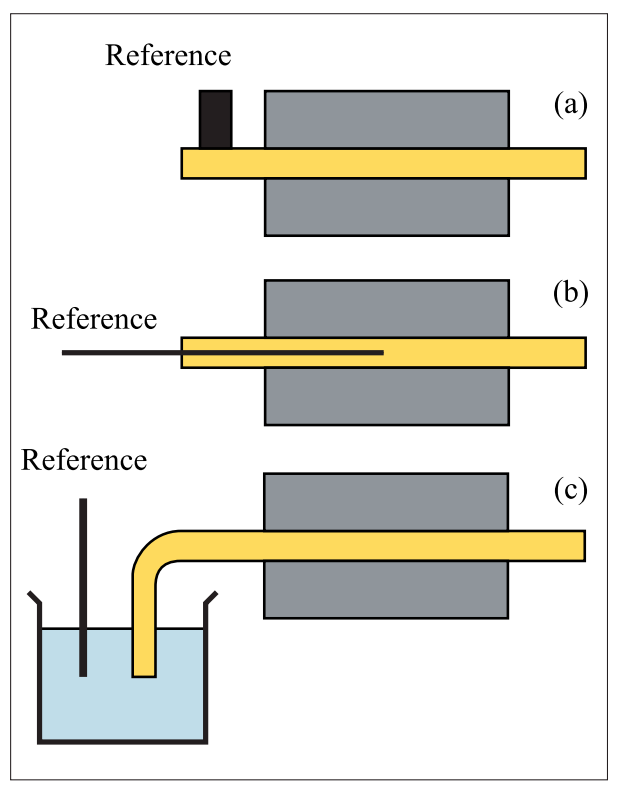

Fig. 1. Three-electrode arrangements applicable to fuel cells; (a) shows a reference placed at the perimeter of the cell; (b) shows the potential probing by placing a wire between the electrodes; (c) shows the incorporation of standard reference electrodes by a electrolyte bridge (only for PEFC)

at the electrodes, due to the implied separation of anodic and cathodic contributions. If the loss processes at one electrode are negligible, the two electrode setup might be chosen, due to the simplicity of the experimental setup. By sufficiently slow variation of the potential, a steady-state current/voltage characteristic of the electrode can be acquired. Although this characteristic is most important from the performance point of view, the extraction of information about contributing processes is limited. Perturbation techniques can be used to separate those processes by their characteristic time constants [2][3]. The three types of perturbations usually applied are shown in Fig. 2. An abrupt potential 'step' can reveal the time-dependent response of the device through the resulting rate adaptation of the current. A current step or abrupt interruption equivalently induces a potential relaxation. Repeated potential ramps, alternately positive- and negative-going, detect any redox (reversible electrochemical reaction) process on the electrode by a hysteresis effect, a technique known as cyclic voltammetry. Finally, a small amplitude sinusoidal modulation of the electrode potential reveals the behaviour of the electrode at the applied frequency. The measured current, in amplitude and phase, is the response of the device at that frequency, inversely related to its response time. It is complementary to the 'step' methods in that time- and frequency-domain effects are Fourier-related. Now a frequency sweep can provide the 'electrochemical impedance spectrum' of the electrode.

The electrochemical diagnostic tools are therefore available to monitor the complete life cycle of a fuel cell device as presented schematically in Fig. 3 (adapted from [4]). Distinction and evaluation of mechanisms characterised by events in the second or millisecond range (e.g. charge transfer and mass transport phenomena) are enabled by impedance spectroscopy and 'step' techniques. Structural and compositional rearrangements on putting a new cell into service, often involving a performance increment as interfaces are activated [5], occur over the first few hours or tens of hours; thereafter there is degradation, to be minimised for an adequate economic in-service performance, over thou- sands of operating hours. These longer term effects are monitored essentially by DC electrochemical methods, although transient techniques such as electrochemical impedance spectroscopy (EIS) can also be applied intermittently to determine evolution of dissipative mechanisms contributing to that longterm degradation.

The active area of fuel cells with technically relevant power output is considerably larger in comparison to cells for materials research or fundamental electrochemical investigations. Inhomogeneities of important parameters (reactant distribution, temperature

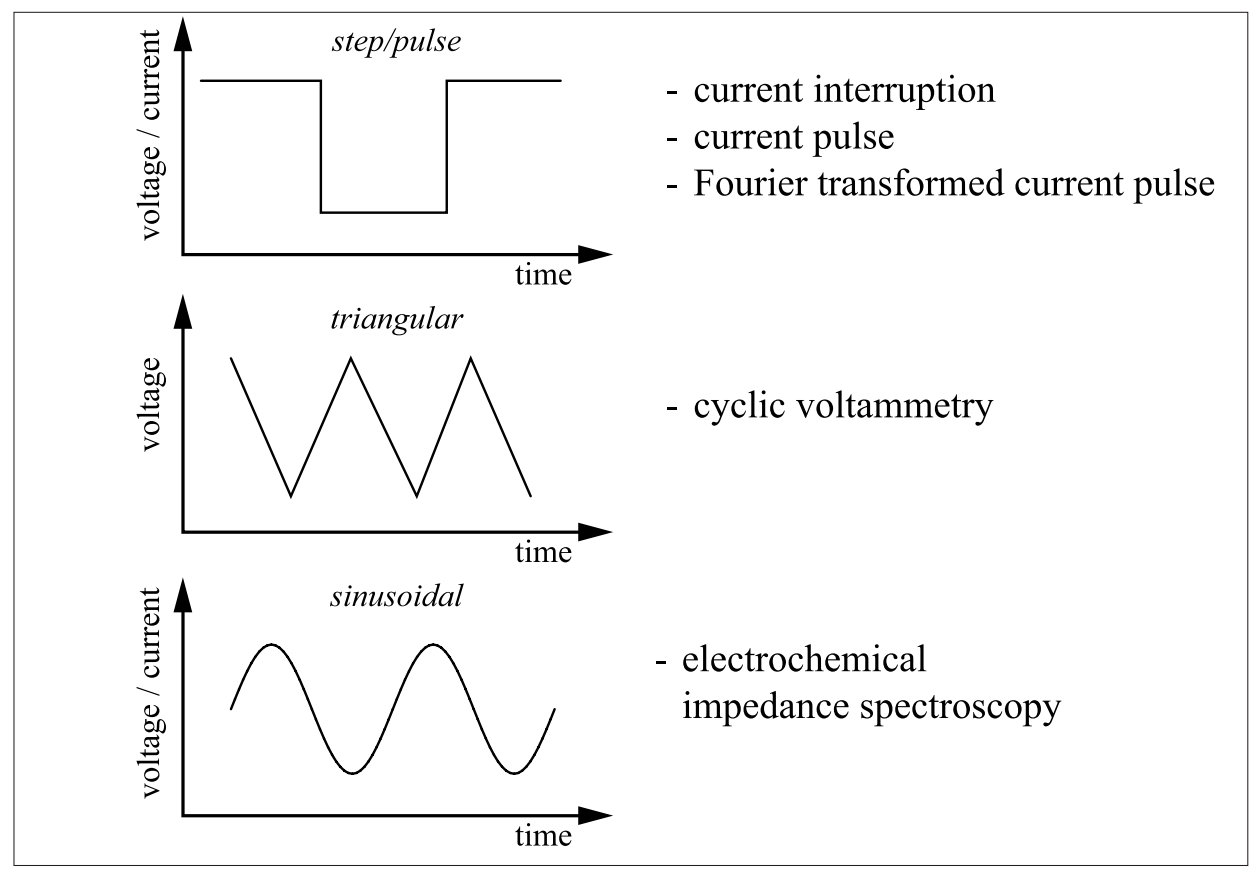

Fig. 2. Perturbation types applicable to fuel cells in order to separate super-imposed processes by their time constants, and analytical methods based on the different types of perturbation

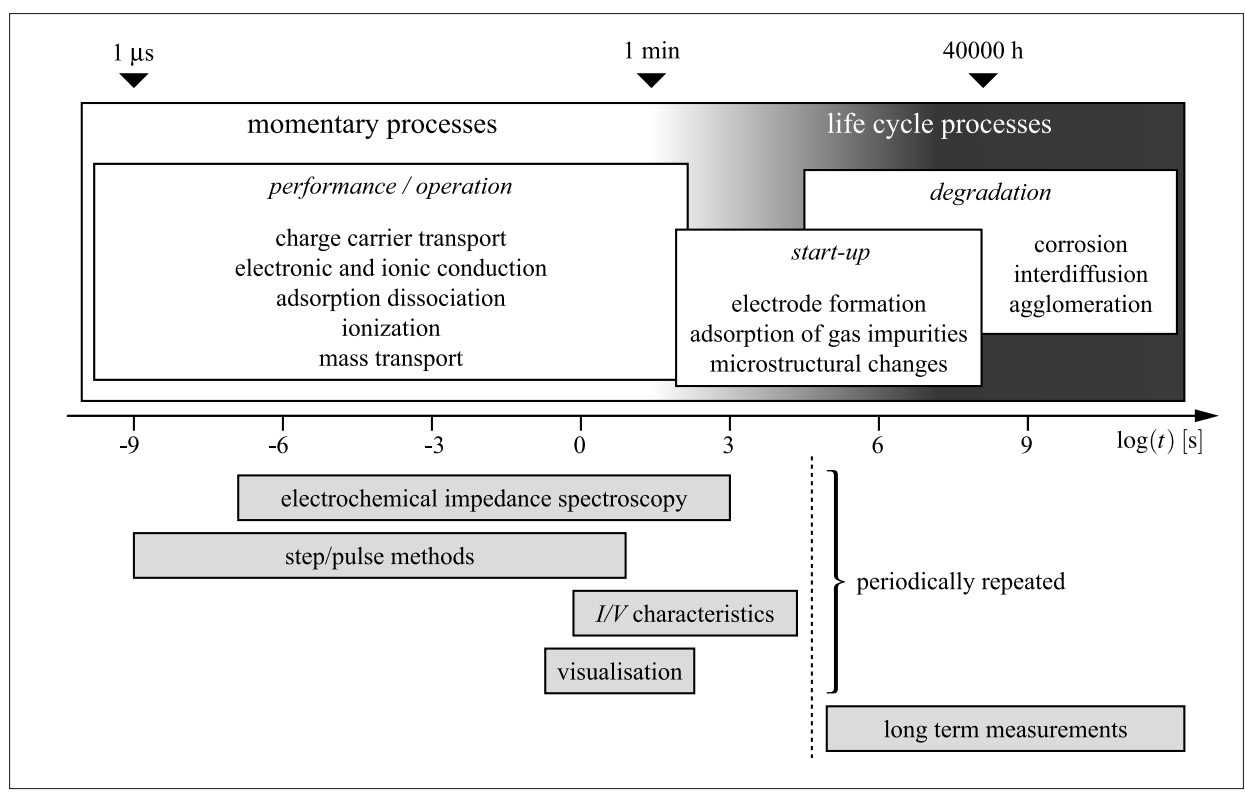

Fig. 3. Relation of measurement techniques to time-domain events in the service life of fuel cells and stacks; adapted from [4] 
gradients, etc.) are unavoidable. Consequently, current is generated inhomogeneously over the cell area. Since inhomogeneities impede the efficiency and performance (scaleup effects), the mapping of local electrochemical performance is an essential diagnostic task on the way to develop and evolve technical-sized systems. Principally this is done by dividing the total area into different regions, and collecting, extracting and measuring current generated in any fractional area separately. Segmented cells have to be used for this, and the challenge is to design measurement systems which do not alter the operation in comparison to the unsegmented sibling. The segmentation of all MEA and cell components would allow the highest accuracy by intrinsically avoiding lateral currents. But, by segmenting current collector and flow field alone a sufficient accuracy can be achieved. Provided the measurement circuit is suitable, even the segmentation of flow fields can be omitted with tolerable errors [6]. The application of many of the above-mentioned electrochemical methods becomes possible, once such a system is realised. However, the measurement of DC-current distribution [6] and spatially resolved impedance spectroscopy [7] are the most prominent techniques.

Electrochemical methods require the interpretation of the experimental findings either analytically or by convergence with modelling results in order to relate them to physicochemical characteristics of the system under investigation. For complicated systems like fuel cells, this often requires several parameters to be considered, and supporting information is needed to validate the conclusions. In consequence, the simultaneous monitoring of characteristics beside the electrochemical behaviour is highly desirable. This includes simple analysis, such as the chemical composition of the effluents, as well as sophisticated experiments, wherein determination of temperature fields, and spatial distribution of species and their state of aggregation are only the most prominent examples.

All electrochemical characterisation procedures are essentially non-destructive in situ methods. Following the electrochemical procedures, conventional materials science post mortem methods (e.g. scanning electron microscopy of surfaces and sections, with elemental resolution) should be applied and the observations correlated with the electrochemistry results in order to develop strategies, including recommended operating procedures, to enhance service life and device efficiency.

\section{Testing Solid Oxide Fuel Cells}

The procedures for electrochemical testing are generic to all types of fuel cells. How- ever, due to the significantly different temperature operating regimes, and consequent effects on electrode mechanisms and kinetics, diagnostic methods do differ. Fig. 4a shows a conventional system for mounting a test cell, by sealing to a refractory tube with glass, then admitting fuel to the internal face. The standard fuel for testing is hydrogen with approx. 3\% water added; this ensures a suitable equilibrium of water with hydrogen and oxygen, determinant of the oxygen effective partial pressure and therefore of the Nernstian potential difference expected across the cell. At a sufficient temperature for thermally activated ionic mobility in the electrolyte, over $600{ }^{\circ} \mathrm{C}$, the device should show an open-circuit potential difference close to the Nernst value; any significant deviation is indicative of a leak or internal short circuit, either in the device itself or in the sealant. For this reason, cells are systematically tested for density and lack of open porosity before mounting; a simple and effective method is to inspect for capillary transport of acetone or similar low-viscosity liquid sprayed on the anode and diffusing to the cathode side. The cell can then be mounted in the test oven and raised to an adequate temperature. Since glass seals are delicate to establish and frequently fracture on cooling, a rapid and effective mounting for intensively used test stations is shown in Fig. 4b [8] where the cell is clamped between two flanges. Ceramic fibre pads distribute fuel and air over the respective electrodes and prevent cross-diffusion between anode and cathode zones.

Of particular importance in thermally activated devices such as solid oxide fuel cells (SOFC) is the accurate measurement of temperature and temperature gradients on the active interfaces, since resistive and other dissipative processes associated with the electrochemical operation of the cell represent a heat source whereby the relevant temperature is significantly higher than that of the test oven. Unless a thermocouple is placed in intimate contact with a fuel cell electrode, a distinctly different temperature may be assumed for the device, with a misinterpretation of its real performance.
While the open-circuit voltage is essentially linked to the structural quality of the electrolyte, the current is determined by charge and mass exchange at the interfaces, essentially therefore by kinetic considerations of electrochemistry, electrocatalysis, adsorption and diffusion of gases. Regarding the current-voltage characteristic, the sharp drop frequently observed for small currents is attributed to activation polarisation, given that the initial production of steam in the anode compartment rapidly displaces the Nernst potential, logarithmically dependent on oxygen partial pressure. After a linear section of the characteristic, which provides the preferred operating point for a practical device, there is a low-voltage current-limited zone due to diffusion losses and depletion of gas supply.

Research and development activities require operation on other fuels, reformed hydrocarbons for example, and even ammonia, as well as investigation of their relative kinetics and anticatalytic poisoning effects due to fuel impurities such as sulphur. This type of activity obviously requires complex supply manifolds, together with gas analysis capability for reaction products.

\section{Testing Polymer Electrolyte Fuel Cells}

Of particular relevance in the testing and operation of polymer electrolyte fuel cell (PEFC) devices is the control of water balance in the cell, as the mobile hydrogen ion is strongly hydrated and therefore the cell current is associated with water transfer from anode to cathode. Insufficient replenishment of anode compartment water leads to an increased polarisation loss, evident in current (or voltage) step as well as impedance spectroscopy results. Excess water impacts similarly on cell performance as a mass transport limitation, particularly by flooding the cathode-side components, such as electrode, GDL, and flow field.

For fundamental investigation of PEFC components and their interaction, the 'mem-

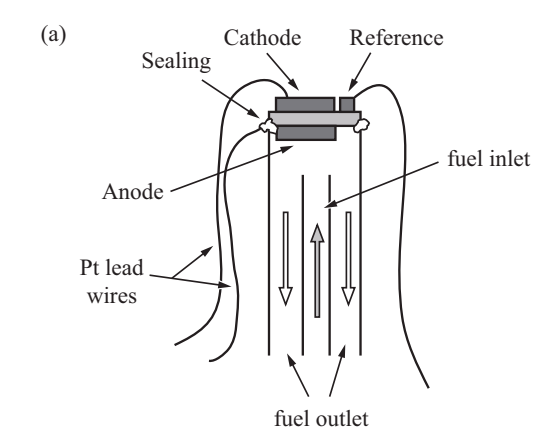

(b)

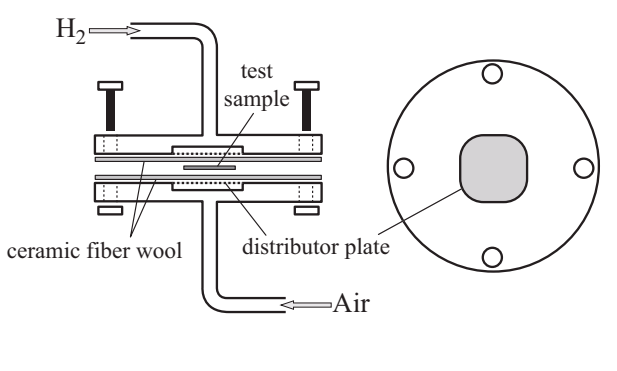

Fig. 4. Mounting systems for solid oxide fuel cell test; (a) sealed with glass on a refractory tube, anode to the interior; (b) rapid test device with mounting flanges and ceramic fibre mats 
brane-electrode-assembly' (MEA) is inserted into a simplified homogeneous working cell - analogous to that used in the SOFC case. Large gas chambers, which replace the usual flow fields of a PEFC, ensure a homogeneous reactant distribution over the active area, and therefore homogeneous current generation. In impedance spectroscopy diagnostics with a two-electrode arrangement, contributions from both anode and cathode processes are not distinguished. The anode contributions of the hydrogen oxidation reaction (HOR) to the total cell impedance are usually less significant, and the measured impedance is attributed to the oxygen reduction reaction (ORR) and the electrolyte. However, at high current densities, the anode contributions must be considered [9][10], requiring the introduction of a three-electrode configuration. With this setup the impedance can be obtained separately for each electrode-electrolyte interface, enabling the study of single electrode characteristics [11]. The features of the anodic and cathodic impedance response are distinguished by measuring against a pseudoreference electrode - placed between the two electrodes, rather than at the perimeter of the cell ( $c f$. Fig. 1b) - and displayed in Fig. 5a. Convergence with results, based on a statespace modelling approach - which is also common in SOFC [12] - incorporating a kinetic model ( $c f$. Fig. 4b), enables the relation of the observed impedance spectra with elementary processes, such as $\mathrm{H}_{2}$ diffusion, adsorption, and charge transfer [13].

PEFCs for research should exhibit active areas of several tens of square centimetres to reduce the influence of edge effects on the cell measurements. In practical applications, fuel cells may even have significantly larger active areas, of the order of several hundreds of square centimetres. As a consequence, inhomogeneities in the current density and the electrode overpotential, due to locally differing reactant and water concentrations and temperature gradients, are incurred. Therefore, locally resolved DC-current measurements in PEFC [6] are a valuable diagnostic tool for mapping inhomogeneous operation, and with the more advanced locally resolved EIS in PEFCs the limiting processes affecting the lateral current distribution can be identified. Both in situ methods making use of a segmented PEFC are presently applied at PSI. The segmented cell (area $29.2 \mathrm{~cm}^{2}$ ) shown in Fig. $6 \mathrm{~b}$ is similar to an ordinary $\mathrm{H}_{2} / \mathrm{O}_{2}$-PEFC with three-fold serpentine flow field. On the cathode side the flow field and current collector are divided into nine electrically isolated segments of equal size. Since the cell is segmented along the gas channel, it is possible to measure the variation of current density and impedance response with changing reactant and water concentration 'along the channel'. Results of locally resolved current density and EIS measurements using this segmented $\mathrm{H}_{2} / \mathrm{O}_{2}$-PEFC are shown in Fig. 6.
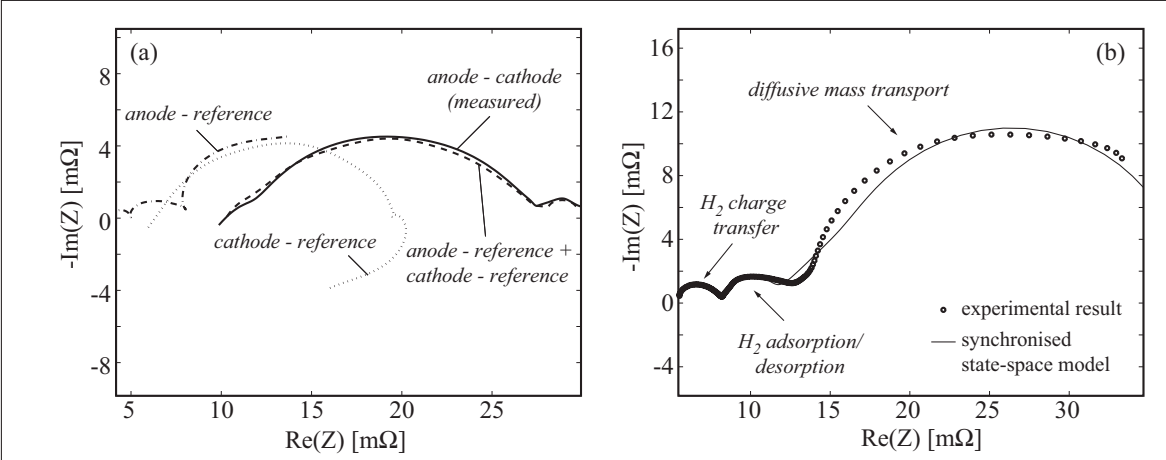

Fig. 5. (a) Impedance spectra of anode/cathode measured with respect to a pseudo reference electrode and between anode and cathode at a current density of $200 \mathrm{~mA} / \mathrm{cm}^{2}$, and (b) comparison of experimental with simulated frequency response of hydrogen oxidation reaction at anodic electrode surface at $500 \mathrm{~mA} / \mathrm{cm}^{2}$; cell temperature is $75^{\circ} \mathrm{C}$; hydrogen rel. humidity is $100 \%$; oxygen rel. humidity is $0 \%$; stoichiometric ratio is 1.5 [13]
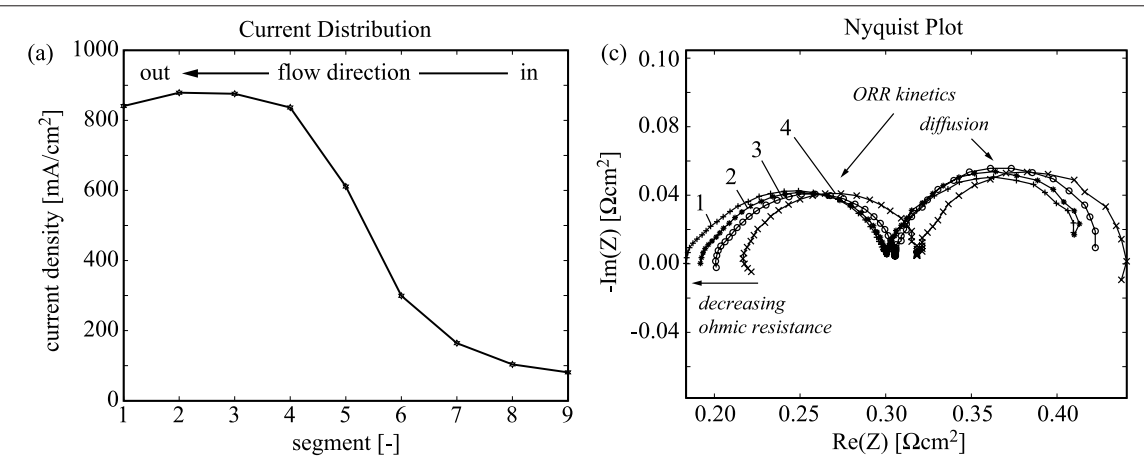

(b)
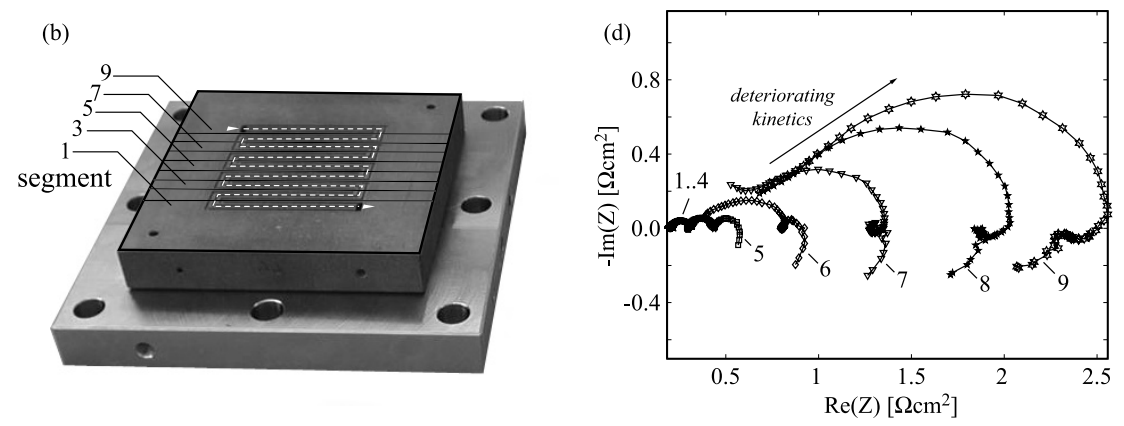

Fig. 6. Segmented $\mathrm{H}_{2} / \mathrm{O}_{2}$-PEFC, DC current distribution (co-flow) and corresponding locally resolved EIS spectra; cell temperature is $70{ }^{\circ} \mathrm{C}$; anodic and cathodic inlet humidity is $50 \%$; stoichiometric ratio is 1.5; cell voltage is $538 \mathrm{mV}$; mean current density is $500 \mathrm{~mA} / \mathrm{cm}^{2}$; Nafion $115 \mathrm{membrane}$; carbon paper based electrodes $0.6 \mathrm{mg} \mathrm{Pt} / \mathrm{cm}^{2}$; frequency range from $0.016 \mathrm{~Hz}$ to $10 \mathrm{kHz}$

The gases are fed to segment 9 at the top and pass in co-flow mode over the active area with the gas outlet at segment 1 at the bottom (Fig. 6b). The benefit of an increasing relative humidity due to the formation of product water along the gas channels is clearly demonstrated. As the humidity increases along the channel from segment 9 to segment 1 , the current density increases as well, although segment 1 shows a decreasing value (Fig. 6a).

The impedance plot of segment 1-4 shows two arcs (Fig. 6c). According to our model the higher frequency arc (left) can be attributed to ORR kinetics, whereas the lower frequency arc (right) is due to diffusion at the anode [7]. Starting at the gas out- let (segment 1), the ohmic resistance increases with decreasing humidity, whereas the charge transfer resistance decreases initially (Fig. 6c). Thus the performance loss of segment 1 , which is lowest in ohmic resistance, can be attributed to flooding at the cathode side and blocking of active sites by liquid water.

With further decreasing humidity and current density along the channel the charge transfer resistance of the ORR strongly increases from segment 5-9 (Fig. 6d). This is accompanied by the formation of an additional higher frequency arc, which might be attributed to HOR kinetics. Furthermore, an inductive behaviour is seen in the lower fre- 


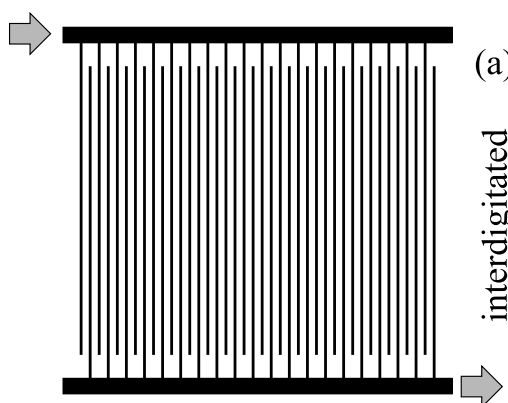

(a)
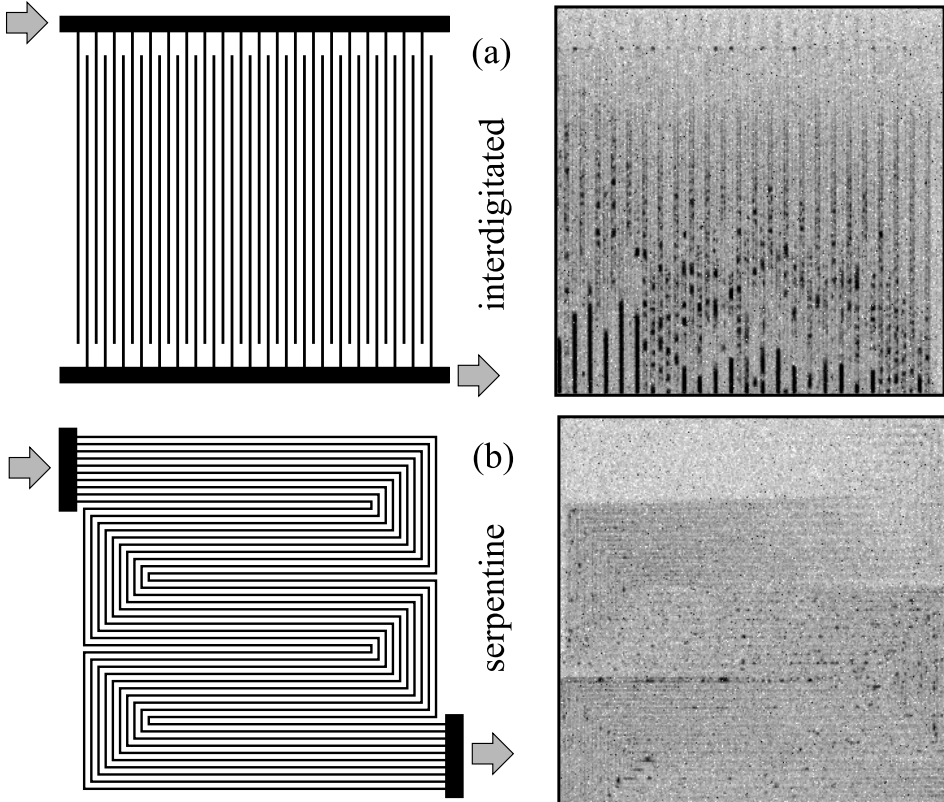

(b)

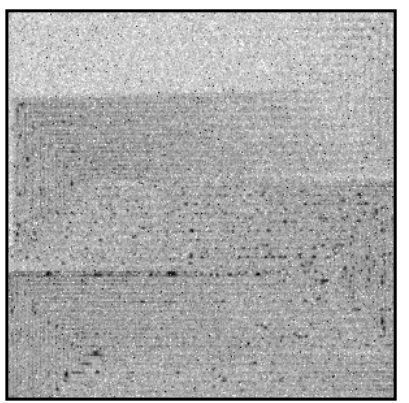

ing device can be imaged. Neutrons are best suited for two-phase flow investigations due to the high cross-section of neutron interaction with the hydrogen nuclei, while interaction with materials such as graphite or aluminium is moderate. The method is available at the neutron radiography station NEUTRA, located at Paul Scherrer Institut's spallation neutron source SINQ. The situation on the cathode side is more critical, with both product water, and water transported with the protons through the membrane requiring removal. Images taken from two cells with different flow distributors are shown in Fig. 7 [16]. While the interdigitated comb-like structure of flow field (a) forces the gases through the porous gas diffusion layer (GDL), the ten-channel serpentine structure of the second flow field (b) provides a continuous connection from inlet to outlet. The cells were operated under the same operating conditions and identical components were used. It is obvious that the interdigitated structure is not able to remove liquid effectively, resulting in accumulations due to gravity at the bottom of the fingers (black spots in the images). These water slugs could not be observed in the serpentine structure, indicating that this geometry removes water more effectively. Nonetheless, liquid accumulation inside the porous structure of the cathodic gas diffusion layer is unavoidable (middle grey shades), resulting in a mass transport limitation as well. This emphasises that improved liquid transport within the porous structures and optimized flow field geometries are equally important to further reduce PEFC propensity to flooding.

\section{Testing Direct Methanol Fuel Cells}

Direct methanol fuel cells (DMFC), where diluted methanol is fed as liquid fuel to the anode compartment, use MEA structures similar to PEFC technology, though obviously the reactions taking place at the anode differ. The oxidation of methanol is a complex reaction. Hence, anodic losses in electrochemical performance are higher than if hydrogen is provided as fuel. To distinguish the different loss processes, a suitable reference electrode is required. This is provided by a hydrogen evolving platinum or 'dynamic' hydrogen electrode (DHE) at the cell perimeter ( $c f$. Fig. 1a). However, placing the reference at the perimeter of the cell implies the necessity of correcting artefacts due to the geometrical arrangement, which is in resemblance to SOFC research. As sketched in Fig. 8a, a small misalignment of the electrodes will cause an asymmetrical opening of the potential field, and the reference probes the potential somewhere inside the electrolyte. In addition, mass transport limitations at the edges of the electrode, differences in the kinetics of the occurring reactions, and inho- 


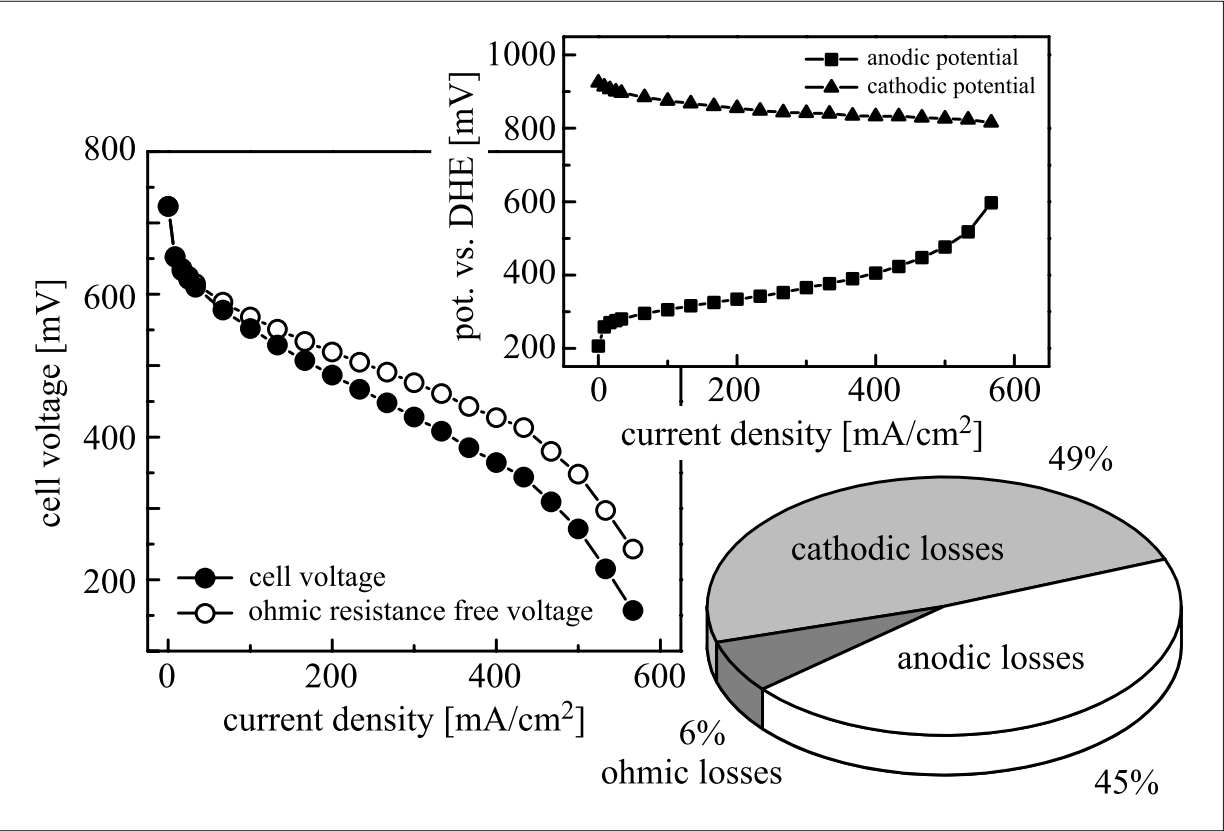

Fig. 9. Resolved polarisation curve of a DMFC operating at $90{ }^{\circ} \mathrm{C}$ cell temperature; anodic supply is $20 \mathrm{ml} / \mathrm{min} 0.5 \mathrm{M}$ methanol; cathodic supply is $500 \mathrm{ml} / \mathrm{min}$ dry air at $90{ }^{\circ} \mathrm{C}$; active area is $30 \mathrm{~cm}^{2}$; the percentages are based on the thermodynamically reversible voltage at standard conditions and correspond to a cell voltage of $400 \mathrm{mV}$

mogeneities of the electrolyte properties might likewise cause an asymmetrical opening of the field. The position of potential probing can be derived from the voltage relaxation after superimposing a fast auxiliary current pulse [17] between anode and cathode. The diagram displayed in Fig. $8 \mathrm{~b}$ shows the voltage relaxation measured between anode and cathode and between anode and DHE, respectively [18]. The discrepancy in step-heights is a measure for the probing point and allows a precise compensation of ohmic contributions. Fig. 9 shows a polarisation curve, whereby losses in a $30 \mathrm{~cm}^{2}$ cell with serpentine flow fields due to anode and cathode kinetics and ohmic resistance are resolved. The inset pie chart shows the contributions to the cell losses at a typical operating point of $400 \mathrm{mV}$. This detailed information is valuable for an adequate interpretation of the polarisation curves and directs research and development by identifying the MEA components and other structures that contribute most to the overall electrochemical performance loss.

As can be seen from Fig. 9, in the high current density range the anodic polarisation increases disproportionately. This is due to a limited transport of methanol to the electrochemically active interface. In order to enhance the mass transport inside the anodic compartment, the accumulation of gaseous carbon dioxide produced in the anodic halfcell reaction has to be understood. Neutron imaging has also been applied to operating DMFC, in order to investigate the accumulation of gaseous $\mathrm{CO}_{2}$ within the anodic flow field [14][19]. As might be expected, an in- crease in the fuel flow rate can reduce the channel volume filled with $\mathrm{CO}_{2}$. However, the comparison with a simultaneous spatially resolved current measurement showed no strict correlation between gas pattern and current generation [20]. This indicates that gaseous $\mathrm{CO}_{2}$ clusters within the porous diffusion media might have higher impact on local performance than those inside the flow field channels.

\section{Conclusions}

Diagnostic methods are essential for progress in fuel cell research and development, as well as to quality control and safety. The separation of losses by perturbation techniques is of highest value while investigating homogeneous working systems. With the development of segmented cell hardware, fundamental experimental analysis can be applied to cells working under technical constraints, and inhomogeneous operation can be evaluated in detail. The requirements of the researcher and the engineer have driven the development of more sophisticated and precise diagnostic methods. This has been illustrated, particularly, with reference to electrochemical testing methods, and indicates the relevance of supplementary analysis, such as visualisation techniques, to validate the conclusions reached in electrochemical testing.

\section{Acknowledgements}

The Federal Office of Energy supported fuel cell research in Switzerland; collaboration with
European Union partners is under the management of the Swiss Federal Office of Education and Science.

Received: November 5, 2004

[1] S.B. Adler, J. Electrochem. Soc. 2002, 149, E166; F. van Heuveln, Thesis, University of Twente (NL), 1997.

[2] C.H. Hamann, W. Vielstich, 'Elektrochemie', Wiley-VCH, 1998, ISBN 3-52727894-x.

[3] A.J. Bard, L.R. Faulkner, 'Electrochemical Methods', Wiley, 2001, ISBN 0-47104372-9.

[4] E. Ivers-Tiffee, A. Weber, H. Schichlein, 'Handbook of Fuel Cells', Vol. 2, 'Electrocatalysis', Eds. W. Vielstich, A. Lamm, H.A. Gasteiger, Wiley (England) 2003, p. 220.

[5] A.J. McEvoy, Solid State Ionics 2000, 135, 331.

[6] A. Geiger, R. Eckl, A. Wokaun, G.G. Scherer, J. Electrochem Soc. 2004, 151, A394.

[7] I. Schneider, H. Kuhn, A. Wokaun, G.G. Scherer, to be published.

[8] J. Van herle, K.R. Thampi, J. Appl. Electrochem. 1994, 25, 970.

[9] B. Andreaus, A.J. McEvoy, G.G. Scherer, Electrochimica Acta 2002, 47, 2223.

[10] B. Andreaus, G.G. Scherer, Solid State Ionics 2004, 168, 311.

[11] H. Kuhn, B. Andreaus, A. Wokaun, G.G. Scherer, PSI Scientific Report, Vol. 5 General Energy, ISSN 1423-7342, 2003, 107.

[12] A. Bieberle, L. J. Gauckler, Solid State Ionics 2002, 146, 23.

[13] H. Kuhn, B. Andreaus, A. Wokaun, G.G. Scherer, Electrochimica Acta, accepted for publication.

[14] A.B. Geiger, A. Tsukada, E. Lehmann, P. Vontobel, A. Wokaun, G.G. Scherer, Fuel Cells 2002, 2, 92.

[15] D. Kramer, J. Zhang, R. Shimoi, E. Lehmann, A. Wokaun, K. Shinohara, G.G. Scherer, Electrochimica Acta, accepted for publication.

[16] D. Kramer, E. Lehmann, P. Vontobel, A. Wokaun, G.G. Scherer, J. Zhang, R. Shimoi, K. Shinohara, PSI Scientific Report, Vol. 5 General Energy, ISSN 1423-7342, 2003, 108.

[17] F.N. Büchi, A. Marek, G.G. Scherer, J. Electrochem. Soc. 1995, 142, 1895.

[18] D. Kramer, S. Loher, G.G. Scherer, PSI Scientific Report, Vol. 5 General Energy, ISSN 1423-7342, 2003, 106.

[19] D. Kramer, E. Lehmann, G. Frei, P. Vontobel, A. Wokaun, G.G. Scherer, Nucl. Instrum. Meth. A, accepted for publication.

[20] D. Kramer, A. Geiger, A. Wokaun, G.G. Scherer, Proc. 2nd European PEFC Forum, Lucerne, ISBN 3-905592-13-4, 2003, 565. 